\title{
On the Hausdorff Analyticity for Quaternion-Valued Functions
}

\section{E. Luna-Elizarrarás, M. Shapiro \& V. Shpakivskyi}

Complex Analysis and Operator Theory

ISSN 1661-8254

Complex Anal. Oper. Theory DOI 10.1007/s11785-018-0856-8

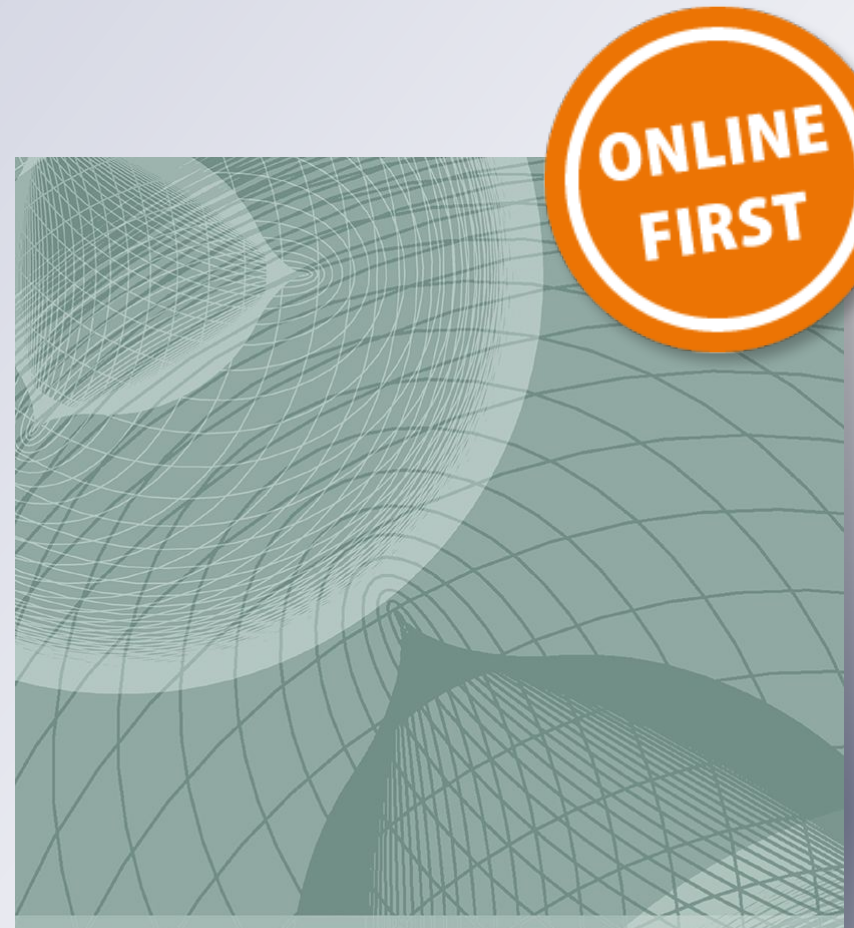

Complex Analysis and Operator Theory

Vol. 7
No. 4

No. 4
pp. $695-1428$

ISSN $1661-8254$

Birkhäuser 
Your article is protected by copyright and all rights are held exclusively by Springer Nature Switzerland AG. This e-offprint is for personal use only and shall not be selfarchived in electronic repositories. If you wish to self-archive your article, please use the accepted manuscript version for posting on your own website. You may further deposit the accepted manuscript version in any repository, provided it is only made publicly available 12 months after official publication or later and provided acknowledgement is given to the original source of publication and a link is inserted to the published article on Springer's website. The link must be accompanied by the following text: "The final publication is available at link.springer.com". 


\title{
On the Hausdorff Analyticity for Quaternion-Valued Functions
}

\author{
M. E. Luna-Elizarrarás ${ }^{1}$ (D) M. Shapiro ${ }^{1} \cdot$ V. Shpakivskyi ${ }^{2}$
}

Received: 14 June 2018 / Accepted: 5 October 2018

(c) Springer Nature Switzerland AG 2018

\begin{abstract}
We consider the concept of the Hausdorff analyticity for functions ranged in real algebras and the corresponding notion of the Hausdorff derivative. Both apply to the real algebra $\mathbb{H}$ of Hamilton's quaternions. The main aim of the work is to compare them with the well-known classes of $\mathbb{H}$-valued functions which have their own definitions of the derivative.
\end{abstract}

Keywords Hausdorff derivative - Quaternions · Quaternionic hyperholomorphic functions · Quaternionic slice regular functions

Mathematics Subject Classification 30G35 · 57R35

\section{Hausdorff Analyticity and Hausdorff Derivative}

\subsection{Introduction}

Hausdorff [10] proposed the definition of an analytic function taking values in a complex associative (commutative or non-commutative) algebra $\mathbb{A}$ with unit. It can be formulated as follows. Let $e_{1}, \ldots, e_{n}$ be a basis of $\mathbb{A}$, let $\eta_{1}, \ldots, \eta_{n}$ be the respective

\section{Communicated by Daniel Aron Alpay.}

$凶 \quad$ M. E. Luna-Elizarrarás

lunae@hit.ac.il

M. Shapiro

shapiro1945@outlook.com

V. Shpakivskyi

shpakivskyi86@gmail.com

1 Holon Institute of Technology, Holon, Israel

2 Institute of Mathematics of the National Academy of Sciences of Ukraine, Kiev, Ukraine 
complex variables and let $\eta:=\sum_{k=1}^{n} \eta_{k} e_{k}$ be the hypercomplex variable, consider an $\mathbb{A}$-valued function

$$
f(\eta)=\sum_{k=1}^{n} f_{k}\left(\eta_{1}, \ldots, \eta_{n}\right) e_{k},
$$

where the components $f_{k}$ are holomorphic functions of $\eta_{1}, \ldots, \eta_{n}$ in the sense of several complex variables theory. In other words, $f$ is a holomorphic mapping from $\mathbb{C}^{n}$ to $\mathbb{C}^{n}$ seen as a hypercomplex function. Its complex differential is defined in the usual way as

$$
\begin{aligned}
d f_{\eta}\left(d \eta_{1}, \ldots, d \eta_{n}\right) & :=\sum_{k=1}^{n} d f_{k}\left(d \eta_{1}, \ldots, d \eta_{n}\right) e_{k} \\
& =\sum_{j, k=1}^{n} \frac{\partial f_{k}}{\partial \eta_{j}}\left(\eta_{1}, \ldots, \eta_{n}\right) d \eta_{j} e_{k}
\end{aligned}
$$

It is assumed tacitly that we deal with a domain in $\mathbb{C}^{n}$ with the coordinates $\eta_{1}, \ldots, \eta_{n}$, and that $d f_{k}$ is an $n$-linear mapping on the complex tangential space to the points of this domain. The function $f$ is called Hausdorff-analytic or $H$-analytic function of the hypercomplex variable $\eta$ if $d f_{\eta}$ is a $\mathbb{C}$-linear homogeneous polynomial of the differential $d \eta:=\sum_{k=1}^{n} d \eta_{k} e_{k}$, i.e.,

$$
d f_{\eta}=\sum_{s=1}^{n^{2}} A_{s}(\eta) d \eta B_{S}(\eta)
$$

where $A_{s}$ and $B_{s}$ are some $\mathbb{A}$-valued functions of the variable $\eta$.

For an $H$-analytic function $f$ the element $f_{H}^{\prime}(\eta):=\sum_{s=1}^{n^{2}} A_{S}(\eta) B_{S}(\eta)$ is called the Hausdorff derivative of the function $f$ at a point $\eta$.

It is worth noting that although the origin of the formula (1.3) is clear: the corresponding antecedents for functions of a real or a complex variable, but at the same time (1.3) is not related explicitly with the Hausdorff derivative and it is not so obvious if both inherit the structures of their antecedents.

There exists a kind of a "real version" of Hausdorff's approach, see [17-19], where $\mathbb{A}$ is a real algebra, $\eta_{1}, \ldots, \eta_{n}$ are real variables, the components $\left\{f_{k}\right\}$ in (1.1) are $\mathbb{R}$-valued functions of $\eta$ and we assume all $f_{k}$ to be $\mathbb{R}$-differentiable at a given point of $\mathbb{R}^{n}$ (the real analyticity is assumed in [19]). In all that follows in this work we deal with such a real approach employing the same terms as above; for instance, $d f_{\eta}$ means the real differential, etc.

\subsection{Relations with some other notions of analyticity}

In the case of a commutative algebra $\mathbb{A}$, the class of $H$-analytic functions coincides with well-known subclasses of differentiable functions. Namely, it is shown in [5] that the class of $H$-analytic functions coincides with the class of Scheffers differentiable 
functions [20] $\left(d f=f^{\prime}(\eta) d \eta\right)$. It follows from the results of paper [21] that the class of $\mathrm{H}$-analytic functions coincides with the classes of functions differentiable in the sense of Gateaux and in the sense of Lorch [11].

Moreover, for a commutative variable $\eta:=\sum_{k=1}^{n} \eta_{k} e_{k}$, where $e_{1}=1$ and $\eta_{k} \in \mathbb{R}$, all mentioned derivatives are equal and

$$
f_{H}^{\prime}(\eta)=\frac{\partial f}{\partial \eta_{1}}(\eta) .
$$

In particular, it is clear that in the algebra of complex numbers $\mathbb{C}$ the $H$-analytic functions coincide with usual holomorphic functions, and for $z:=x+i y$ we have: $f_{H}^{\prime}(z)=f^{\prime}(z)=\frac{\partial f}{\partial x}(z)$.

\subsection{Quaternionic functions and H-analyticity}

We now realize the Hausdorff approach for functions with values in the skew-field of (real) quaternions $\mathbb{H}$. We use the notations $e_{1}=1$ and $e_{2}, e_{3}, e_{4}$ for the canonical imaginary units, thus $\left\{e_{1}=1, e_{2}=i, e_{3}=j, e_{4}=k\right\}$ is a basis of $\mathbb{H}=\mathbb{R}^{4}$.

Let $\Omega$ be a domain in $\mathbb{H}$. Consider a function $f: \Omega \rightarrow \mathbb{H}$ of the variable $x=$ $x_{1} e_{1}+x_{2} e_{2}+x_{3} e_{3}+x_{4} e_{4}$ given as

$$
f(x)=\sum_{k=1}^{4} f_{k}(x) e_{k} .
$$

Definition 1.1 The function $f: \Omega \rightarrow \mathbb{H}$ of the form (1.5) is $H$-analytic in the domain $\Omega$, if $f$ is analytic by Hausdorff at every point $x \in \Omega$, i.e., if the real-valued components $f_{k}$ are real differentiable functions of four real variables $x_{1}, x_{2}, x_{3}, x_{4}$, and the differential $d f_{x}=\sum_{k=1}^{4} d f_{k}\left(x_{1}, x_{2}, x_{3}, x_{4}\right) e_{k}$ is an $\mathbb{R}$-linear homogeneous polynomial of the differential $d x:=d x_{1} e_{1}+d x_{2} e_{2}+d x_{3} e_{3}+d x_{4} e_{4}$, i.e.,

$$
d f_{x}=\sum_{s=1}^{16} A_{s}(x) d x B_{s}(x)
$$

where $A_{s}$ and $B_{s}$ are some $\mathbb{H}$-valued functions of the variable $x$.

In this case, for any $x \in \Omega$ the quaternion

$$
f_{H}^{\prime}(x):=\sum_{s=1}^{16} A_{s}(x) B_{S}(x)
$$

is the Hausdorff derivative ( $H$-derivative) of the function $f$ at the point $x$.

Denote by $\mathfrak{M}_{H}(\Omega)$ the set of all quaternion-valued $H$-analytic functions in the domain $\Omega$. 


\subsection{Examples of quaternionic $\mathrm{H}$-analytic functions}

Let us consider some examples of quaternionic $H$-analytic functions and compute their $H$-derivatives.

Example Let $f_{n}(x)=x^{n}$ for $n \in \mathbb{N}$.

If $n=1$ then $f_{1}(x)=x$ and $d f_{1}=d x=1 \cdot d x \cdot 1$ thus $f_{1}$ is $H$-analytic and its derivative is

$$
f_{1, H}^{\prime}(x)=1 \text { for all } x
$$

If $n=2$ then $f_{2}(x)=x^{2}$ and $d f_{2}(x)=d\left(x^{2}\right)=x \cdot d x+d x \cdot x$. Therefore, $f_{2}$ is $H$-analytic and

$$
f_{2, H}^{\prime}(x)=\left(x^{2}\right)_{H}^{\prime}=x \cdot 1+1 \cdot x=2 x
$$

In the same way for the function $f_{3}(x)=x^{3}$ we have:

$$
d\left(x^{3}\right)=d\left(x^{2}\right) \cdot x+x^{2} \cdot d x=x \cdot d x \cdot x+d x \cdot x^{2}+x^{2} \cdot d x .
$$

Therefore $f_{3}$ is also $H$-analytic and its derivative is

$$
f_{3, H}^{\prime}(x)=\left(x^{3}\right)_{H}^{\prime}=x \cdot x+1 \cdot x^{2}+x^{2} \cdot 1=3 x^{2} .
$$

By induction $f_{n}$ is $H$-analytic for all $n \in \mathbb{N}$ with the derivative $f_{n, H}^{\prime}(x)=\left(x^{n}\right)_{H}^{\prime}=$ $n x^{n-1}$. Clearly $x^{n} \cdot a$ and $a \cdot x^{n}$ are also $H$-analytic for all $a \in \mathbb{H}$ and their $H$-derivatives are $n \cdot x^{n-1} \cdot a$ and $a \cdot n \cdot x^{n-1}$. It is obvious that the polynomials $\sum_{n=0}^{m} x^{n} a_{n}, \sum_{n=0}^{m} a_{n} x^{n}$ are $H$-analytic and

$$
\left(\sum_{n=0}^{m} x^{n} a_{n}\right)_{H}^{\prime}=\sum_{n=1}^{m} n x^{n-1} a_{n}, \quad\left(\sum_{n=0}^{m} a_{n} x^{n}\right)_{H}^{\prime}=\sum_{n=1}^{m} n a_{n} x^{n-1} .
$$

Example Since the quaternionic multiplication is non-commutative, more sophisticated polynomials should be considered. First, let $f(x)=x a x b x$, where $a, b \in \mathbb{H}$, then

$$
d(x a x b x)=d x \cdot a x b x+x a \cdot d x \cdot b x+x a x b \cdot d x .
$$

Therefore, the function $f$ is $H$-analytic and

$$
f_{H}^{\prime}(x)=a x b x+x a b x+x a x b .
$$

In the same way, it can be shown that any general quaternionic polynomial:

$$
p_{n}(x)=\sum_{k=0}^{n} M_{k}(x)
$$


is $H$-analytic where the monomial $M_{k}$ is defined by $M_{k}:=\sum_{\ell=1}^{m} a_{k, \ell, 1} x a_{k, \ell, 2} x$. $\ldots \cdot x a_{k, \ell, k+1}, m$ is some natural number, and $a_{k, \ell, p} \in \mathbb{H}$.

\subsection{The H-derivative of quaternionic functions}

The peculiarities of the skew-field $\mathbb{H}$ allow one to describe all the quaternionic $H$ analytic functions and their $H$-derivatives.

Theorem 1.2 Any quaternionic function with real differentiable components is $H$ analytic.

Proof It is known that

$$
\begin{aligned}
& x_{1}=\frac{1}{4}\left(e_{1} x e_{1}-e_{2} x e_{2}-e_{3} x e_{3}-e_{4} x e_{4}\right), \\
& x_{2}=\frac{1}{4}\left(-e_{1} x e_{2}-e_{2} x e_{1}+e_{3} x e_{4}-e_{4} x e_{3}\right), \\
& x_{3}=\frac{1}{4}\left(-e_{1} x e_{3}-e_{3} x e_{1}-e_{2} x e_{4}+e_{4} x e_{2}\right), \\
& x_{4}=\frac{1}{4}\left(-e_{1} x e_{4}-e_{4} x e_{1}+e_{2} x e_{3}-e_{3} x e_{2}\right),
\end{aligned}
$$

which implies the same kind of formulas for the differentials:

$$
\begin{aligned}
& d x_{1}=\frac{1}{4}\left(e_{1} d x e_{1}-e_{2} d x e_{2}-e_{3} d x e_{3}-e_{4} d x e_{4}\right), \\
& d x_{2}=\frac{1}{4}\left(-e_{1} d x e_{2}-e_{2} d x e_{1}+e_{3} d x e_{4}-e_{4} d x e_{3}\right), \\
& d x_{3}=\frac{1}{4}\left(-e_{1} d x e_{3}-e_{3} d x e_{1}-e_{2} d x e_{4}+e_{4} d x e_{2}\right), \\
& d x_{4}=\frac{1}{4}\left(-e_{1} d x e_{4}-e_{4} d x e_{1}+e_{2} d x e_{3}-e_{3} d x e_{2}\right)
\end{aligned}
$$

Take now the differential $d f$ and use (1.8)-(1.11):

$$
\begin{aligned}
d f= & \sum_{k=1}^{4} \frac{\partial f}{\partial x_{k}} d x_{k} \\
= & \frac{\partial f}{\partial x_{1}} \cdot \frac{1}{4}\left(e_{1} d x e_{1}-e_{2} d x e_{2}-e_{3} d x e_{3}-e_{4} d x e_{4}\right) \\
& +\frac{\partial f}{\partial x_{2}} \cdot \frac{1}{4}\left(-e_{1} d x e_{2}-e_{2} d x e_{1}+e_{3} d x e_{4}-e_{4} d x e_{3}\right) \\
& +\frac{\partial f}{\partial x_{3}} \cdot \frac{1}{4}\left(-e_{1} d x e_{3}-e_{3} d x e_{1}-e_{2} d x e_{4}+e_{4} d x e_{2}\right) \\
& +\frac{\partial f}{\partial x_{4}} \cdot \frac{1}{4}\left(-e_{1} d x e_{4}-e_{4} d x e_{1}+e_{2} d x e_{3}-e_{3} d x e_{2}\right)
\end{aligned}
$$


We abbreviate $\partial_{k} f:=\frac{\partial f}{\partial x_{k}}(x)$, then

$$
\begin{aligned}
d f= & \frac{1}{4} \cdot\left(\left(\partial_{1} f e_{1} d x e_{1}-\partial_{2} f e_{2} d x e_{1}-\partial_{3} f e_{3} d x e_{1}-\partial_{4} f e_{4} d x e_{1}\right)\right. \\
& +\left(-\partial_{1} f e_{2} d x e_{2}-\partial_{2} f e_{1} d x e_{2}+\partial_{3} f e_{4} d x e_{2}-\partial_{4} f e_{3} d x e_{2}\right) \\
& +\left(-\partial_{1} f e_{3} d x e_{3}-\partial_{2} f e_{4} d x e_{3}-\partial_{3} f e_{1} d x e_{3}+\partial_{4} f e_{2} d x e_{3}\right) \\
& \left.+\left(-\partial_{1} f e_{4} d x e_{4}+\partial_{2} f e_{3} d x e_{4}-\partial_{3} f e_{2} d x e_{4}-\partial_{4} f e_{1} d x e_{4}\right)\right) \\
= & \frac{1}{4} \cdot\left(\left(\partial_{1} f e_{1}-\partial_{2} f e_{2}-\partial_{3} f e_{3}-\partial_{4} f e_{4}\right) d x e_{1}\right. \\
& +\left(-\partial_{1} f e_{2}-\partial_{2} f e_{1}+\partial_{3} f e_{4}-\partial_{4} f e_{3}\right) d x e_{2} \\
& +\left(-\partial_{1} f e_{3}-\partial_{2} f e_{4}-\partial_{3} f e_{1}+\partial_{4} f e_{2}\right) d x e_{3} \\
& \left.+\left(-\partial_{1} f e_{4}+\partial_{2} f e_{3}-\partial_{3} f e_{2}-\partial_{4} f e_{1}\right) d x e_{4}\right) \\
= & \frac{1}{4} \cdot\left(\overline{\mathcal{D}}_{F, r}[f] e_{1} d x e_{1}-\widehat{\mathcal{D}}_{F, r}[f] e_{2} d x e_{2}\right. \\
& \left.-\widetilde{\mathcal{D}}_{F, r}[f] e_{3} d x e_{3}-\widetilde{\mathcal{D}}_{F, r}[f] e_{4} d x e_{4}\right)
\end{aligned}
$$

with

$$
\begin{aligned}
& \overline{\mathcal{D}}_{F, r}[f]:=\partial_{1} f e_{1}-\partial_{2} f e_{2}-\partial_{3} f e_{3}-\partial_{4} f e_{4} ; \\
& \widehat{\mathcal{D}}_{F, r}[f]:=\partial_{1} f-\partial_{2} f e_{2}+\partial_{3} f e_{3}+\partial_{4} f e_{4} ; \\
& \widetilde{\mathcal{D}}_{F, r}[f]:=\partial_{1} f+\partial_{2} f e_{2}-\partial_{3} f e_{3}+\partial_{4} f e_{4} ; \\
& \widetilde{\widetilde{D}}_{F, r}[f]:=\partial_{1} f+\partial_{2} f e_{2}+\partial_{3} f e_{3}-\partial_{4} f e_{4} .
\end{aligned}
$$

The theorem is proved.

It is worth mentioning that the operator $\overline{\mathcal{D}}_{F, r}$ is the quaternionic conjugate of the famous (right-hand side) Fueter operator acting on the $C^{1}$-functions by the formula $\mathcal{D}_{F, r}[f]:=\partial_{1} f+\partial_{2} f e_{2}+\partial_{3} f e_{3}+\partial_{4} f e_{4}$; both play a crucial role in quaternionic analysis, see Sect. 3 in this work. Meanwhile the rest three operators are "partial quaternionic conjugations" of $\mathcal{D}_{F, r}$ playing the role of the hyperderivatives for the respective classes of hyperholomorphic functions.

Using definition (1.7), we obtain:

$$
f_{H}^{\prime}(x)=\frac{1}{4}\left(\overline{\mathcal{D}}_{F, r}[f]+\widehat{\mathcal{D}}_{F, r}[f]+\widetilde{\mathcal{D}}_{F, r}[f]+\widetilde{\mathcal{D}}_{F, r}[f]\right)=\frac{\partial f}{\partial x_{1}}
$$

From Theorem 1.2 the next example follows immediately.

Example Given a quaternionic convergent series

$$
\sum_{n=0}^{\infty} x^{n} a_{n}
$$


then in the domain of its convergence it is an $H$-analytic function, the same for $\sum_{n=0}^{\infty} a_{n} x^{n}$. Furthermore, any general convergent quaternionic series of the form

$$
q(x)=\sum_{k=0}^{\infty} M_{k}(x)
$$

is an $H$-analytic function in the domain of its convergence.

Now we show that the series (1.13) can be differentiated term-wise in the sense of Hausdorff.

Theorem 1.3 If a series (1.13) converges in the ball $B:=\{x \in \mathbb{H}:|x|<R\}$, then its sum is an $H$-analytic function in $B$ and this series can be differentiated term-wise in the sense of Hausdorff derivative:

$$
\left(\sum_{n=0}^{\infty} x^{n} a_{n}\right)_{H}^{\prime}=\sum_{n=1}^{\infty} n x^{n-1} a_{n}
$$

Proof By Theorem 1.2 the series (1.13) is an $H$-analytic function. It means that the function (1.13) has a Hausdorff derivative.

The series (1.13) can be represented as

$$
\sum_{n=0}^{\infty} x^{n} a_{n}=f_{1} e_{1}+f_{2} e_{2}+f_{3} e_{3}+f_{4} e_{4}
$$

where $f_{k}\left(x_{1}, x_{2}, x_{3}, x_{4}\right)=\sum_{m, n, p, q=0}^{\infty} \alpha_{m, n, p, q}^{(k)} x_{1}^{m} x_{2}^{n} x_{3}^{p} x_{4}^{q}, k=1,2,3,4$, are real-analytic functions on the ball $B$. As a consequence, the series for $f_{k}$ can be differentiated term-wise by $x_{1}$ on the interval $(-R, R)$, with $x_{2}, x_{3}, x_{4}$ restricted in such a way that $x \in B$. In turn, it means that the left-hand side of the equality (1.15) can be differentiated term-wise by $x_{1}$. By formula (1.12) we have

$$
\left(\sum_{n=0}^{\infty} x^{n} a_{n}\right)_{H}^{\prime}=\frac{\partial}{\partial x_{1}} \sum_{n=0}^{\infty} x^{n} a_{n}=\sum_{n=0}^{\infty} \frac{\partial}{\partial x_{1}}\left(x^{n} a_{n}\right)=\sum_{n=1}^{\infty} n x^{n-1} a_{n} .
$$

The theorem is proved.

As a consequence, if we introduce the elementary functions $\cos x, \sin x$, exp $x$ as the sums of the respective power series then these functions become $H$-analytic with

$$
(\cos x)_{H}^{\prime}=-\sin x, \quad(\sin x)_{H}^{\prime}=\cos x, \quad(\exp x)_{H}^{\prime}=\exp x
$$




\section{A Relation Between $\boldsymbol{H}$-Analyticity and the "Naive" Quaternionic Differentiability}

By "naive" approach we mean direct analogs of the corresponding definitions for real and complex functions which are based on the study of the differential quotient of a given function. The non-commutativity of quaternionic multiplication leads to the two of them:

$$
\begin{aligned}
h & \mapsto h^{-1} \Delta f_{x}(h), \\
h & \mapsto \Delta f_{x}(h) h^{-1},
\end{aligned}
$$

where for a given quaternionic function $f: \Omega \rightarrow \mathbb{H}$ one sets

$$
\Delta f_{x}(h):=f(x+h)-f(x) .
$$

By the properties of quaternionic conjugation one has also:

$$
h^{-1} \Delta f_{x}(h)=\overline{\Delta \bar{f}_{x}(h) \bar{h}^{-1}} \text {. }
$$

Thus, each of the cases reduces to its counterpart, and we'll treat mostly one of them.

Definition 2.1 (Quaternionic derivability) Assume that (2.1) has a limit if $h \rightarrow 0$, then we say that $f$ is left-derivable at $x$ and the limit itself is called the left-hand side derivative of $f$ at $x$ and is denoted as

$$
f(x):=\lim _{\substack{h \rightarrow 0 \\ x+h \in \Omega}} h^{-1} \Delta f_{x}(h) .
$$

Similarly for the right-hand side derivative.

Definition 2.2 (Quaternionic differentiability) Let $h \rightarrow 0$; if there exists a constant $B_{x} \in \mathbb{H}$ such that

$$
\Delta f_{x}(h)=h B_{x}+o(h),
$$

then $f$ is called left-differentiable at $x$. Similarly for the right-differentiability.

Both definitions hold simultaneously only. Formula (2.3) allows us to relate the left-hand side and the right-hand side situations. The next observation is worth noting.

The function $f: x \mapsto a x+b(a, b \in \mathbb{H})$ is right-differentiable in the whole $\mathbb{H}$, while the function $g: x \mapsto x a+b$ is left-differentiable which seems to be quite natural. Recall that as we know these functions are $H$-analytic also. A quadratic polynomial is also $H$-analytic, what about its "naive" differentiability?

Consider $\alpha: x \mapsto x^{2}$, then

$$
\Delta \alpha_{x}(h)=x h+h x+h^{2}
$$


and for $x \notin \mathbb{R}$ the principal part, $x h+h x$, is in general neither quaternionic left-, nor quaternionic right-linear, which means that $\alpha$ is not derivable in $\mathbb{H} \backslash \mathbb{R}$ ! It is instructive to compare this reasoning with the definition of $H$-analyticity.

The next theorem describes the general situation.

Theorem 2.3 (See, e.g., [12]) Let $f: \Omega \rightarrow \mathbb{H}$ be quaternionic left-differentiable throughout the (connected) domain $\Omega$ and let $g: \Omega \rightarrow \mathbb{H}$ be quaternionic rightdifferentiable. Then there exist two quaternionic constants, a and $b$, such that

$$
f(x)=a+x b \quad \forall x \in \Omega,
$$

and there exist two quaternionic constants $p$ and $q$ such that

$$
g(x)=p+q x \quad \forall x \in \Omega
$$

In [12] one can find more details about the topic.

Corollary 2.4 The set of quaternionic left-differentiable and of quaternionic rightdifferentiable functions in $\Omega$ are subsets of the set $\mathfrak{M}_{H}(\Omega)$. Moreover,

$$
f_{H}^{\prime}(x)={ }^{\prime} f(x)=b, \quad g_{H}^{\prime}(x)=g^{\prime}(x)=q .
$$

\section{A Relation Between $\mathrm{H}$-Analyticity and Fueter-Hyperholomorphy}

As "naive" quaternionic differentiability does not produce an interesting theory one may ask if another approach would lead to a more interesting developments.

It is widely known since 1920s (Gr. K. Moisil, N. Teodorescu, R. Fueter) that a rich quaternionic function theory arises if one generalizes directly the idea of complex Cauchy-Riemann operators.

Let $\Omega$ be a domain in $\mathbb{R}^{4}$, consider $C^{1}(\Omega, \mathbb{H})$ on which the (Fueter) operator $\mathcal{D}_{F}:=\sum_{\ell=1}^{4} e_{\ell} \frac{\partial}{\partial x_{\ell}}$ is well-defined. Its structure is evidently similar to that of the complex Cauchy-Riemann operator $\frac{\partial}{\partial \bar{z}}$. We call the functions in $\mathfrak{M}_{F}(\Omega):=\operatorname{ker} \mathcal{D}_{F}$ hyperholomorphic (other names used are monogenic, regular, hyperanalytic, etc.). That is, the Fueter hyperholomorphic functions satisfy

$$
\mathcal{D}_{F}[f](x):=\sum_{k=1}^{4} e_{k} \frac{\partial f}{\partial x_{k}}=0 .
$$

Beginning with the works of Moisil and Teodorescu, then of Fueter, the functions in $\mathfrak{M}_{F}(\Omega)$ have been considered to be a good generalization of holomorphic functions in one complex variable. They possess many properties deeply similar to their complex one-dimensional antecedents: Cauchy integral theorem; hyperholomorphic Cauchy kernel generating Cauchy integral representation for hyperholomorphic functions and hyperholomorphic Cauchy transform; the Taylor and Laurent series, and many others. Besides, quaternionic hyperholomorphic functions have found an ample range of 
applications in mathematics, physics, etc. Thus one can hope to have an analogue of the complex derivative for quaternionic hyperholomorphic functions.

We begin with the equality which plays a crucial role in complex analysis of one variable:

$$
d f_{z_{0}}=\frac{\partial f}{\partial z}\left(z_{0}\right) d z+\frac{\partial f}{\partial \bar{z}}\left(z_{0}\right) d \bar{z}
$$

with

$$
\frac{\partial f}{\partial z}=\frac{1}{2}\left(\frac{\partial}{\partial x}-i \frac{\partial}{\partial y}\right), \quad \frac{\partial f}{\partial \bar{z}}=\frac{1}{2}\left(\frac{\partial}{\partial x}+i \frac{\partial}{\partial y}\right)
$$

The equality (3.2) has the two quaternionic analogues, a left-hand side and a righthand side ones:

$$
\begin{aligned}
& d\left(\sigma^{(2)} \cdot f\right)=\frac{1}{2}\left(\sigma^{(3)} \cdot \overline{\mathcal{D}}_{F}[f]-\overline{\sigma^{(3)}} \cdot \mathcal{D}_{F}[f]\right), \\
& d\left(g \cdot \sigma^{(2)}\right)=\frac{1}{2}\left(\overline{\mathcal{D}}_{F, r}[g] \cdot \sigma^{(3)}-\mathcal{D}_{F, r}[g] \cdot \overline{\sigma^{(3)}}\right),
\end{aligned}
$$

where $f, g \in C^{1}(\Omega, \mathbb{H})$,

$$
\begin{aligned}
\sigma^{(2)}= & i d x_{2} \wedge d x_{3}+j d x_{3} \wedge d x_{1}+k d x_{1} \wedge d x_{2}, \\
\sigma^{(3)}= & d x_{1} \wedge d x_{2} \wedge d x_{3}-i d x_{0} \wedge d x_{2} \wedge d x_{3} \\
& +j d x_{0} \wedge d x_{1} \wedge d x_{3}-k d x_{0} \wedge d x_{1} \wedge d x_{2},
\end{aligned}
$$

and $\mathcal{D}_{F, r}$ means the action of the operator $\mathcal{D}_{F}$ on the right, $\overline{\mathcal{D}}_{F}$ is the quaternionic conjugate of $\mathcal{D}_{F}$.

The following analogies are clear:

$$
\begin{array}{ll}
\mathcal{D}_{F} \longleftrightarrow \frac{\partial}{\partial \bar{z}} ; & \overline{\mathcal{D}}_{F} \longleftrightarrow \frac{\partial}{\partial z} ; \\
\sigma^{(3)} \longleftrightarrow d z ; & \overline{\sigma^{(3)}} \longleftrightarrow d \bar{z}
\end{array}
$$

A geometric explanation of the meaning of the differential form $\sigma^{(3)}$ is worth noting: if $\Gamma$ is a smooth hypersurface in $\mathbb{R}^{4}, \tau \in \Gamma, \mathbf{n}(\tau)=\left(n_{0}, n_{1}, n_{2}, n_{3}\right)$ is the outward pointing normal vector then $\sigma^{(3)} \mid \Gamma=n(\tau) d S$ where $n(\tau)=\sum_{k=1}^{4} e_{k} n_{k}, d S$ is the differential form of the hypersurface in $\mathbb{R}^{4}$. The same happens in the complex situation: $d z=-i d \ell$.

Equation (3.5) hints also at the relation between $\overline{\mathcal{D}}_{F}$ and the future derivative.

Definition 3.1 (Hyperdifferentiability for the Fueter hyperholomorphy) A function $f \in C^{1}(\Omega, \mathbb{H})$ is called hyperdifferentiable if there exists $A_{x} \in \mathbb{H}$ such that

$$
d\left(\sigma^{(2)} f\right)=\sigma^{(3)} \cdot A_{x}
$$


Thus the idea of a "proportionality coefficient" between the two differential forms is realized although, of course, in a much more sophisticated mode: now we deal with differential forms of degree 3 . The latter is because of the hyperdimension in $\mathbb{R}^{4}$, which gives also a hint on how to define the appropriate "increments of the function and of its argument". Given $x^{0} \in \Omega$, let

$$
\Pi:=\left\{x^{0}+\sum_{k=1}^{3} h_{k} t_{k} \in \mathbb{R}^{4} \mid\left(t_{1}, t_{2}, t_{3}\right) \in[0,1]^{3}\right\}
$$

be a parallelepiped with vertex $x^{0}$ and let

$$
\partial \Pi:=\left\{x^{0}+\sum_{k=1}^{3} h_{k} t_{k} \in \mathbb{R}^{4} \mid\left(t_{1}, t_{2}, t_{3}\right) \in \partial\left([0,1]^{3}\right)\right\}
$$

be its boundary. In the complex case $\Pi=\left[z_{0}, z\right]$, a straight segment, and $\partial \Pi=\left\{z_{0}, z\right\}$, a set consisting of two points.

Definition 3.2 (Hyperderivability for the Fueter operator)

- Given a parallelepiped $\Pi$ with vertex $x^{0}$, the integral

$$
\int_{\partial \Pi} \sigma^{(2)} f(x)
$$

is called the increment of the function $f$ at $x^{0}$, and the integral

$$
\int_{\Pi} \sigma^{(3)}
$$

is called the increment of the argument.

- Given a sequence $\left\{\Pi_{n}\right\}_{n \in \mathbb{N}}$ of parallelepipeds with vertex $x^{0}$ and such that

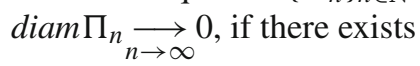

$$
\lim _{n \rightarrow \infty}\left(\left(\int_{\Pi_{n}} \sigma^{(3)}\right)^{-1} \cdot \int_{\partial \Pi_{n}} \sigma^{(2)} f(x)\right)
$$

then $f$ is called hyperderivable at $x^{0}$ and the limit itself, denoted by ' $f\left(x^{0}\right)$, is called the hyperderivative of $f$ at $x^{0}$.

The relations between the three notions are described as follows.

Theorem 3.3 $f \in \mathfrak{M}_{F}(\Omega)$ if and only if $f$ is hyperdifferentiable. And for such functions

$$
A_{x}=\frac{1}{2} \overline{\mathcal{D}}_{F}[f](x)
$$


Theorem 3.4 If $f \in \mathfrak{M}\left(x^{0}\right)$ then $f$ is hyperderivable at $x^{0}$ and

$$
A_{x^{0}}={ }^{\prime} f\left(x^{0}\right)=\frac{1}{2} \overline{\mathcal{D}}_{F}\left(x^{0}\right) .
$$

For a function $f \in \mathfrak{M}_{F}(\Omega)$ the hyperderivative' $f$ can be calculated by the formula

$$
{ }^{\prime} f(x)=\frac{1}{2} \overline{\mathcal{D}}_{F}[f](x)=\frac{1}{2}\left(e_{1} \frac{\partial f}{\partial x_{1}}-\sum_{k=2}^{4} e_{k} \frac{\partial f}{\partial x_{k}}\right) .
$$

Since by definition $\mathfrak{M}_{F}(\Omega) \subset C^{1}(\Omega)$, and $H$-analytic functions have $\mathbb{R}$ differentiable real-valued components, then it makes sense to compare the sets $\mathfrak{M}_{H}(\Omega)$ and $\mathfrak{M}_{F}(\Omega)$ under the same smoothness conditions.

Theorem 3.5 On the class $\mathcal{C}^{1}(\Omega)$ we have the strict inclusion $\mathfrak{M}_{F}(\Omega) \subset \mathfrak{M}_{H}(\Omega)$. Moreover,

$$
{ }^{\prime} f(x)=f_{H}^{\prime}(x)=\frac{\partial f}{\partial x_{1}} .
$$

Proof Firstly, by Theorem 1.2, $\mathcal{C}^{1}(\Omega) \subset \mathfrak{M}_{H}(\Omega)$. But, except the condition $\mathfrak{M}_{F}(\Omega) \subset$ $\mathcal{C}^{1}(\Omega)$, Fueter-hyperholomorphic functions contain an additional condition (3.1). Therefore, $\mathfrak{M}_{F}(\Omega) \subset \mathfrak{M}_{H}(\Omega)$.

Now we prove the equality (3.7). Since the condition $f \in \mathfrak{M}_{F}(\Omega)$ is equivalent to $\sum_{k=1}^{4} e_{k} \frac{\partial f}{\partial x_{k}}=0$, then

$$
-\frac{1}{2} \sum_{k=1}^{4} e_{k} \frac{\partial f}{\partial x_{k}}=0
$$

To both sides of the equality (3.8) we add the expression $\frac{\partial f}{\partial x_{1}}$ :

$$
\frac{1}{2}\left(e_{1} \frac{\partial f}{\partial x_{1}}-\sum_{k=2}^{4} e_{k} \frac{\partial f}{\partial x_{k}}\right)=\frac{\partial f}{\partial x_{1}}
$$

The left-hand side of (3.9) is equal to ' $f(x)$, and we have ' $f(x)=\frac{\partial f}{\partial x_{1}}$. Taking into account Eq. (1.12), we obtain the equality (3.7).

Remark 3.6 We will have an analogous result if we consider the right-hand side Fueter operator, i.e.,

$$
\sum_{k=1}^{4} \frac{\partial f}{\partial x_{k}} e_{k}=0
$$

More details, together with proofs and references can be found in [12,16,22]. 


\section{A Relation Between $H$-Analyticity and $M T$-Hyperholomorphy}

We have seen that, somewhat paradoxically, the class of quaternionic functions with real-differentiable components coincides with the class of quaternionic $\mathrm{H}$ analytic in $\Omega \subset \mathbb{R}^{4}$ functions. Thus, the class of quaternionic functions with real-differentiable components possesses intrinsically a number of properties, possesses an additional structure; in particular, such functions have the $H$-derivative. This remains true for Fueter-hyperholomorphic functions which is a proper subset of quaternionic $H$-analytic functions, but the former has its own intrinsic structure which is not, generally speaking, a restriction of that of the $H$-analyticity: there are many quaternionic $C^{1}$ functions which are not Fueter-hyperholomorphic. Besides, a Fueterhyperholomorphic function has the derivative whose definition is deeply different with that of the $H$-analyticity although their values coincide.

In this section we consider another well-known class of $\mathbb{H}$-valued functions which can be seen as a subclass of Fueter-hyperholomorphic functions.

Definition 4.1 A function $f: \Omega \subset \mathbb{R}^{3} \rightarrow \mathbb{H}$ of class $C^{1}(\Omega)$ is called MoisilTeodorescu-hyperholomorphic ( $M T$-hyperholomorphic) if at each point of $\Omega$ the function $f$ satisfies the equality

$$
\mathcal{D}_{M T}[f](x):=\sum_{k=2}^{4} e_{k} \frac{\partial f}{\partial x_{k}}(x)=0 .
$$

Denote by $\mathfrak{M}_{M T}(\Omega)$ the set of all $M T$-hyperholomorphic functions in the domain $\Omega$.

We can think of $\mathcal{D}_{M T}$ as the operator $\mathcal{D}_{F}$ but acting on functions which do not depend on the variable $x_{1}$; then for $f \in \mathfrak{M}_{M T}(\Omega)$ there holds: $f \in \mathfrak{M}_{F}(\mathbb{R} \times \Omega)$ since $\mathcal{D}_{F}[f]=\frac{\partial f}{\partial x_{1}}+\mathcal{D}_{M T}[f]=0+0=0$. Hence $f$ has the Fueter-derivative $\overline{\mathcal{D}}_{F}[f]=\frac{\partial f}{\partial x_{1}}=0$. Moreover, it is clear that we have the inclusion $\mathfrak{M}_{M T} \subset \mathfrak{M}_{H}$ and

$$
f_{M T}^{\prime}(x)=f_{H}^{\prime}(x)=0
$$

It turns out that the $M T$-hyperholomorphic functions can be endowed with another derivative which possesses the same properties as the Fueter one; what is more, there exist three ways of doing this.

Consider

$$
\mathcal{D}_{M T}^{i}[f](x):=e_{2} \mathcal{D}_{M T}[f](x)=\frac{\partial f}{\partial x_{2}}-e_{4} \frac{\partial f}{\partial x_{3}}+e_{3} \frac{\partial f}{\partial x_{4}}=0 .
$$

Clearly, the set of solutions of Eq. (4.1) coincides with the set $\mathfrak{M}_{M T}$ but the structure of the operator $\mathcal{D}_{M T}^{i}$ is as that of $\mathcal{D}_{F}$ and not as that of $\mathcal{D}_{M T}$ which allows to repeat the constructions of the previous Sect. 3 obtaining the definition and the properties of the new derivative $f_{M T, i}^{\prime}$ for any $M T$-hyperholomorphic function $f$. In particular, we have the following formula for the $i$-hyperderivative (see [12, p. 537]): 


$$
f_{M T, i}^{\prime}=\frac{1}{2} \overline{\mathcal{D}_{M T}^{i}}[f](x)=\frac{1}{2}\left(\frac{\partial f}{\partial x_{2}}+e_{4} \frac{\partial f}{\partial x_{3}}-e_{3} \frac{\partial f}{\partial x_{4}}\right) .
$$

Consider the variable $x:=x_{2} e_{2}+x_{3} e_{3}+x_{4} e_{4}=e_{2}\left(x_{2}-x_{3} e_{4}+x_{4} e_{3}\right)$. Denote by $\tilde{x}$ the variable $x_{2}-x_{3} e_{4}+x_{4} e_{3}$.

Now we will consider functions of $\operatorname{ker} \mathcal{D}_{M T}^{i}$ of the variable $\tilde{x}$. Let a function $f: \widetilde{\Omega}=$ $-e_{2} \Omega \longrightarrow \mathbb{H}$ belongs to $\operatorname{ker} \mathcal{D}_{M T}^{i}(\widetilde{\Omega})=\mathfrak{M}_{M T}(\widetilde{\Omega})$. Note that the $i-$ hyperderivative of $f(\widetilde{x}) \in \mathfrak{M}_{M T}(\widetilde{\Omega})$ is defined by equality (4.2).

The following relations are easy to verify:

$$
\begin{aligned}
& x_{2}=\frac{1}{2}\left(e_{1} \tilde{x} e_{1}-e_{2} \tilde{x} e_{2}\right), \\
& x_{3}=\frac{1}{2}\left(e_{4} \tilde{x} e_{1}-e_{2} \tilde{x} e_{3}\right), \\
& x_{4}=-\frac{1}{2}\left(e_{3} \tilde{x} e_{1}+e_{2} \tilde{x} e_{4}\right) .
\end{aligned}
$$

Using the relations (4.3), similarly to the proof of Theorem 1.2 the formula for the $H$-derivative can be proved:

$$
f_{H}^{\prime}(\tilde{x})=\frac{\partial f}{\partial x_{2}} .
$$

Theorem 4.2 On the class $\mathcal{C}^{1}(\widetilde{\Omega})$ we have the inclusion $\mathfrak{M}_{M T}(\widetilde{\Omega}) \subset \mathfrak{M}_{H}(\widetilde{\Omega})$. Moreover,

$$
f_{M T, i}^{\prime}(\tilde{x})=f_{H}^{\prime}(\widetilde{x})=\frac{\partial f}{\partial x_{2}} .
$$

Proof The inclusion $\mathfrak{M}_{M T}(\widetilde{\Omega}) \subset \mathfrak{M}_{H}(\widetilde{\Omega})$ is obvious.

Now we prove the equality (4.5). Since

$$
f \in \mathfrak{M}_{M T}(\widetilde{\Omega}) \Longleftrightarrow \frac{\partial f}{\partial x_{2}}-e_{4} \frac{\partial f}{\partial x_{3}}+e_{3} \frac{\partial f}{\partial x_{4}}=0,
$$

then

$$
-\frac{1}{2}\left(\frac{\partial f}{\partial x_{2}}-e_{4} \frac{\partial f}{\partial x_{3}}+e_{3} \frac{\partial f}{\partial x_{4}}\right)=0 .
$$

To both sides of equality (4.6) we add the expression $\frac{\partial f}{\partial x_{2}}$ :

$$
\frac{1}{2}\left(\frac{\partial f}{\partial x_{2}}+e_{4} \frac{\partial f}{\partial x_{3}}-e_{3} \frac{\partial f}{\partial x_{4}}\right)=\frac{\partial f}{\partial x_{2}} .
$$

The left-hand side of (4.7) is equal to $f_{M T, i}^{\prime}(\tilde{x})$, and we have $f_{M T, i}^{\prime}(\tilde{x})=\frac{\partial f}{\partial x_{2}}$. Taking into account the equality (4.4), we obtain the equality (4.5).

There are two obvious analogues of the formula (4.1):

$$
\mathcal{D}_{M T}^{j}[f](x):=e_{3} \mathcal{D}_{M T}[f](x)=e_{4} \frac{\partial f}{\partial x_{2}}+\frac{\partial f}{\partial x_{3}}-e_{2} \frac{\partial f}{\partial x_{4}}=0,
$$




$$
\mathcal{D}_{M T}^{k}[f](x):=e_{4} \mathcal{D}_{M T}[f](x)=-e_{3} \frac{\partial f}{\partial x_{2}}+e_{2} \frac{\partial f}{\partial x_{3}}+\frac{\partial f}{\partial x_{4}}=0 .
$$

They lead to the definitions of the two more derivatives for $M T$-hyperholomorphic functions together with analogues of all the conclusions above.

One can find more details about $M T$-hyperholomorphy in [12,14].

\section{A Relation Between $\mathrm{H}$-Analyticity and Clifford Analysis for Quaternion-Valued Functions}

Let $\mathcal{C} \ell_{0, m}$ denote the real Clifford algebra with imaginary units $e_{1}, e_{2}, \ldots, e_{m}$ and the real unit $e_{0}=1$. Of course, the parameter $m$ runs over all natural numbers and when $m=2$ one gets $\mathcal{C} \ell_{0,2}=\mathbb{H}$. In Clifford analysis we have an analogue of the Fueter operator which is usually called the Cauchy-Riemann operator and it together with its Clifford conjugate are given by

$$
\mathcal{D}_{C R}:=\sum_{\ell=0}^{m} e_{\ell} \frac{\partial}{\partial x_{\ell}}, \quad \overline{\mathcal{D}}_{C R}:=\sum_{\ell=0}^{m} \bar{e}_{\ell} \frac{\partial}{\partial x_{\ell}} .
$$

The function theory for $\operatorname{ker} \mathcal{D}_{C R}$ is Clifford analysis, and since $\mathcal{C} \ell_{0,2}=\mathbb{H}$ then one can expect that for $m=2$ the Cauchy-Riemann operator coincides with the Fueter operator which is not the case: $\mathcal{D}_{C R}:=\sum_{\ell=0}^{2} e_{\ell} \frac{\partial}{\partial x_{\ell}}$, that is, we deal with an operator acting on functions of three real variables. The fine point here is that $\mathcal{D}_{C R}$ involves only the imaginary units $e_{1}=i, e_{2}=j$ but not their product $k=i j$ as the Fueter operator does. For this reason the function theory for $\mathcal{D}_{C R}$ and $m=2$ is called the Clifford analysis for $\mathbb{H}$-valued functions. It turns out that the above presented scheme of constructing the hyper-derivative for the Fueter-hyperholomorphic functions can be followed here as well if one selects properly the analogues of the differential forms $\sigma^{(3)}$ and $\sigma^{(2)}$. In this case they are:

$$
\begin{aligned}
\sigma_{x} & :=d \hat{x}_{0}-e_{1} d \hat{x}_{1}+e_{2} d \hat{x}_{2}, \\
\tau_{x} & :=-e_{1} d \widehat{x}_{0,1}+e_{2} d \widehat{x}_{0,2}-e_{2} d \widehat{x}_{0,2},
\end{aligned}
$$

where $d \widehat{x}_{k}$ and $d \widehat{x}_{0, k}$ are obtained from $d V:=d x_{0} \wedge d x_{1} \wedge d x_{2}$ by omitting the respective factors. An important property of $\sigma_{x}$ is that for its restriction onto a smooth, 2-dimensional surface in $\mathbb{R}^{3}$ there holds: $\sigma_{x}=n_{x} d S_{x}$, where $\vec{n}_{x}=\left(n_{0}, n_{1}, n_{2}\right)$ is an outward pointing normal vector to the surface, $n_{x}:=\sum_{k=0}^{2} n_{k} e_{k} ; d S_{x}$ is the differential form of the 2-dimensional surface area in $\mathbb{R}^{3}$.

Now, for $f \in C^{1}\left(\Omega, \mathcal{C} \ell_{0,2}=\mathbb{H}\right)$ there holds:

$$
d\left(\tau_{x} f(x)\right)=\frac{1}{2} \sigma_{x} \overline{\mathcal{D}}_{C R}[f](x)-\frac{1}{2} \bar{\sigma}_{x} \mathcal{D}_{C R}[f](x),
$$

which is again a crucial fact since it allows to introduce the notions of hyperdifferentiability and hyperderivability in the context of Clifford analysis for the 
Cauchy-Riemann operator. One can find a description of the details and fine points in [2,13,15], see also [9].

There is an analogue of the Moisil-Theodorescu operator in Clifford analysis setting as well which is called the Dirac operator. Let now $\Omega$ be a domain in $\mathbb{R}^{m}$. On the set $C^{1}\left(\Omega ; C \ell_{0, m}\right)$ the Dirac operator is defined by

$$
\mathcal{D}_{\text {Dir }}:=\sum_{\ell=1}^{m} e_{\ell} \frac{\partial}{\partial x_{\ell}}
$$

Note that for the formal Clifford conjugate of $\mathcal{D}_{\text {Dir }}$ one has that $\overline{\mathcal{D}}_{\text {Dir }}=-\mathcal{D}_{\text {Dir }}$. Thus, inflating $\Omega$ up to a domain $\widetilde{\Omega} \subset \mathbb{R}^{m+1}$ we can write

$$
\mathcal{D}_{C R}=\sum_{\ell=0}^{m} e_{\ell} \frac{\partial}{\partial x_{\ell}}=\frac{\partial}{\partial x_{0}}+\mathcal{D}_{D i r}
$$

and hence we can identify a function $f \in k e r \mathcal{D}_{\text {Dir }}$ with the function $\tilde{f} \in \operatorname{ker} \mathcal{D}_{C R}$ by

$$
\tilde{f}\left(x_{0}, x_{1}, x_{2}, \ldots, x_{m}\right):=f\left(x_{1}, x_{2}, \ldots, x_{m}\right) \text { for any } x_{0}
$$

Hence any function in $k e r \mathcal{D}_{\text {Dir }}$ has the hyperderivative in the sense of the CauchyRiemann operator but it is identically zero in $\Omega$ :

$$
\tilde{f}\left(x_{0}, x^{0}\right)=\frac{1}{2} \overline{\mathcal{D}}_{C R}[\tilde{f}]\left(x_{0}, x^{0}\right)=-\mathcal{D}_{\operatorname{Dir}}[f]\left(x_{1}, x_{2}, \ldots, x_{m}\right)=0
$$

What happens for $m=2$, i.e., for $\mathcal{C} \ell_{0,2}=\mathbb{H}$ ? We have:

$$
\begin{aligned}
\mathcal{D}_{\text {Dir }} & =\sum_{\ell=1}^{2} e_{\ell} \frac{\partial}{\partial x_{\ell}}=i \frac{\partial}{\partial x_{1}}+j \frac{\partial}{\partial x_{2}} \\
& =i\left(\frac{\partial}{\partial x_{1}}-k \frac{\partial}{\partial x_{2}}\right)=j\left(\frac{\partial}{\partial x_{2}}+k \frac{\partial}{\partial x_{1}}\right)
\end{aligned}
$$

which means that the function theory for the quaternionic Dirac operator reduces to holomorphic, or anti-holomorphic, functions of one complex variable.

Similarly to the proof of Theorem 3.5 the next result can be proved.

Theorem 5.1 On the class $\mathcal{C}^{1}(\Omega)$ we have the inclusion $\operatorname{ker} \mathcal{D}_{C R}(\Omega) \subset \mathfrak{M}_{H}(\Omega)$. Moreover,

$$
{ }^{\prime} f_{C R}(x)=f_{H}^{\prime}(x)=\frac{\partial f}{\partial x_{0}} .
$$




\section{A Relation Between $\mathrm{H}$-Analyticity and Slice-Regularity}

The theory of the so-called slice-regular functions has been introduced by Gentili and Struppa [6] on the basis of development of Cullen's idea [4]. The last decade the theory of slice-regular functions has been very actively developing (see, e.g., [1,3,8]). We will consider quaternionic slice-regular functions in the ball $B:=\{x \in \mathbb{H}:|x|<R\}$.

Since the algebra of quaternions is not commutative, it is natural that there exist left and right slice-regular functions. The class of all left slice-regular functions $f$ : $B \rightarrow \mathbb{H}$ in the domain $B$ coincides with the convergent in $B$ power series $\sum_{n=0}^{\infty} x^{n} a_{n}$, $a_{n} \in \mathbb{H}$ (see e.g., Theorem 2.7 in [7], or [8, p. 15], or Theorem 6.1.5 in [1]). Moreover, the left slice-derivative ' $f_{s}(x)$ of a power series $\sum_{n=0}^{\infty} x^{n} a_{n}$ equals $\sum_{n=1}^{\infty} n x^{n-1} a_{n}$. Analogously, the class of all right slice-regular functions $f: B \rightarrow \mathbb{H}$ in the domain $B$ coincides with convergent power series of the form $\sum_{n=0}^{\infty} a_{n} x^{n}, a_{n} \in \mathbb{H}$, and the right slice-derivative $f_{s}^{\prime}(x)$ of a power series $\sum_{n=0}^{\infty} a_{n} x^{n}$ equals $\sum_{n=1}^{\infty} n a_{n} x^{n-1}$. We consider only the case of left slice-regular functions.

Theorem 6.1 The set of (left or right) slice-regular functions $f: B \rightarrow \mathbb{H}$ in $B$ is a subset of the set $\mathfrak{M}_{H}(B)$. Moreover,

$$
{ }^{\prime} f_{s}(x)=f_{H}^{\prime}(x)=\frac{\partial f}{\partial x_{1}}
$$

for left slice-regular functions and

$$
f_{s}^{\prime}(x)=f_{H}^{\prime}(x)=\frac{\partial f}{\partial x_{1}}
$$

for right slice-regular functions.

Proof Consider the case of left slice-regular functions. In Example 1.5 we show that every left slice-regular function $f(x)=\sum_{n=0}^{\infty} x^{n} a_{n}$ is $H$-analytic in the ball of convergence of this series. Moreover, by Theorem 1.3 the $H$-derivative $f_{H}^{\prime}(x)$ coincides with the left slice-derivative ' $f_{S}(x)$. The case of right slice-regular functions can be dealt with analogously. The Theorem is proved.

At the same time, there exist $H$-analytic functions that are neither left slice-regular, nor right slice-regular (see Example 1.4).

\section{References}

1. Alpay, D., Colombo, F., Sabadini, I.: Slice Hyperholomorphic Schur Analysis. Birkhäuser, Basel (2016)

2. Bory-Reyes, J., Shapiro, M.: Clifford analysis versus its quaternionic counterparts. Math. Methods Appl. Sci. 33(9), 1089-1101 (2010)

3. Colombo, F., Sabadini, I., Struppa, D.C.: Noncommutative Functional Calculus: Theory and Applications of Slice Hyperholomorphic Functions. Progress in Mathematics. Birkhäuser, Basel (2011)

4. Cullen, C.G.: An integral theorem for analytic intrinsic functions on quaternions. Duke Math. J. 32, 139-148 (1965) 
5. Degtereva, M.: To the question of the construction of a theory of analytic functions in linear algebras. Dokl. Akad. Nauk SSSR 61(1), 13-15 (1948). (in Russian)

6. Gentili, G., Struppa, D.C.: A new approach to Cullen-regular functions of a quaternionic variable. Comptes Rendus Mathematique 342(10), 741-744 (2006)

7. Gentili, G., Struppa, D.: A new theory of regular functions of a quaternionic variable. Adv. Math. 216, 279-301 (2007)

8. Gentili, G., Stoppato, G., Struppa, D.: Regular Functions of a Quaternionic Variable. Springer, Berlin (2013)

9. Gürlebeck, K., Malonek, H.R.: A hypercomplex derivative of monogenic functions in $\mathbb{R}^{n+1}$ and its applications. Complex Var. 39, 199-228 (1999)

10. Hausdorff, F.: Zur Theorie der Systeme complexer Zahlen. Leipziger Berichte 52, 43-61 (1900)

11. Lorch, E.R.: The theory of analytic runction in normed abelian vector rings. Trans. Am. Math. Soc. 54, 414-425 (1943)

12. Luna-Elizarrarás, M.E., Shapiro, M.: A survey on the (hyper-) derivatives in complex, quaternionic and Clifford analysis. Milan J. Math. 79(2), 521-542 (2011)

13. Luna-Elizarrarás, M.E., Macias-Cedeño, M.A., Shapiro, M.: Hyperderivatives in Clifford analysis and some applications to the Cliffordian Cauchy-type integrals. In: Sabadini, I., Shapiro, M., Sommen, F. (eds.) Hypercomplex Analysis. Series: Trends in Mathematics, pp. 221-234 (2009)

14. Luna-Elizarrarás, M.E., Macías-Cedeño, M.A., Shapiro, M.: On the hyperderivatives of MoisilThéodoresco hyperholomorphic functions. In: Sabadini, I., Sommen, F. (eds.) Hypercomplex Analysis and Applications. Series: Trends in Mathematics, pp. 181-194 (2011)

15. Luna-Elizarrarás, M.E., Macías-Cedeño, M.A., Shapiro, M.: On the hyperderivatives of Dirachyperholomorphic functions of Clifford analysis. Oper. Theory Adv. Appl. 220, 179-195 (2012)

16. Mitelman, I.M., Shapiro, M.: Differentiation of the Martinelli-Bochner integrals and the notion of hyperderivability. Math. Nachr. 172, 211-238 (1995)

17. Portman, W.O.: A derivative for Hausdorff-analytic functions. Proc. Am. Math. Soc. V 10, 101-105 (1959)

18. Rinehart, R.F., Wilson, J.C.: Two types of differentiability of functions on algebras. Rend. Circ. Matem. Palermo II(11), 204-216 (1962)

19. Ringleb, F.: Beiträge zur funktionentheorie in hyperkomplexen systemen I. Rend. Circ. Mat. Palermo 57(1), 311-340 (1933)

20. Scheffers, G.: Verallgemeinerung der Grundlagen der gewohnlich complexen Funktionen. Ber. Verh. Sachs. Akad. Wiss. Leipzig Mat.-Phys. K1. 45, 828-848 (1893)

21. Shpakivskyi, V.S.: Constructive description of monogenic functions in a finite-dimensional commutative associative algebra. Adv. Pure Appl. Math. 7(1), 63-75 (2016)

22. Sudbery, A.: Quaternionic analysis. Math. Proc. Camb. Philos. Soc. 85, 199-225 (1979) 